\section{Trickreicher Staphylococcus aureus}

$\mathrm{N}$ ur wenige Keime können mehr Schaden anrichten als Staphylococcus aureus. Infektionen mit S. aureus können trotz Einsatz von Antibiotika chronische und immer wieder aufflammende Verläufe nehmen. Eine Forschergruppe um Dr. Bettina Löffler, Münster, hat untersucht, mit welchen Strategien es S. aureus immer wieder gelingt, einer vollständigen Eliminierung zu entgehen [EMBO Molecul Med 2011; 3: 129-41].

Das Ergebnis: Die Bakterien nehmen vorübergehend ein weniger aggressives Erscheinungsbild an, so genannte Small
Colony Variants (SCV). Nicht nur in klinischen Proben werden diese Keime oft nicht entdeckt, auch das körpereigene Immunsystem übersieht die "Schläfer“. Auf diese Weise entkommt ein Teil der S.-aureus-Population sowohl den Attacken des Immunsystems als auch der Vernichtung durch Antibiotika.

Nach einer gewissen Zeit können sich die verbliebenen Bakterien in den Wildtyp zurückverwandeln und die Infektion von neuem entfachen. Dabei ist es typisch, dass die Rückfälle Monate oder auch Jahre nach einer Akutbehandlung und

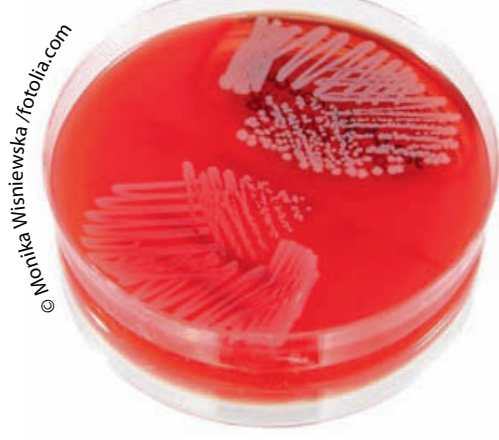

scheinbarer Ausheilung der Krankheit auftreten können.

„Künftige Therapien sollten genau auf diese Fähigkeit des Umschaltens der Bakterien abzielen und verhindern, dass sie sich in den Wirtszellen verstecken“, fordert Löffler.

\section{Impressum}

\section{hautnah dermatologie}

27. Jahrgang,ISSN 0938-0221

Postvertriebsstück B 9868, Entgelt bezahlt.

\section{Redaktion}

Markus Seidl (es)

Dr. (Univ. Perugia) Ulrich Schneider (uls)

Tel. (0 89) 2030 43-14 07, Fax - 1400

E-Mail: ulrich.schneider@springer.com

Assistenz: Christine Hecke

Tel. (0 89) 2030 43-14 02

\section{Beirat}

Prof. Dr. med. D. Abeck, München; Prof. Dr. med. R. Bauer, Bonn; Dr. med. H. Beierdörffer, Hamburg; Dr. med. H. Bresser, München; Prof. Dr. med. E. Christophers, Kiel; PD Dr. med.

Dorothee Dill-Müller, Homburg; Prof. Dr. med. M. Hundeiker, Münster; Dr. med. M. von Ingersleben, Puchheim; Dr. med. Th. Jansen, Essen;

Prof. Dr. med. Ursula Kaben, Kessin; Dr. med. H. Lieske, Hamburg; Dr. med. C. Meisel, Nürnberg; Dr. med. U. Mutschler, Hildesheim; Prof. Dr. med. R. Niedner, Potsdam; Dr. med. J. A. v. Preyss, Hamburg; Prof. Dr. med. U. Reinhold, Bonn; Prof. Dr. med. Dr. phil. J. Ring, München; Prof. Dr. med. B. Th. Rohde, Hamburg; Dr. med. H. Schulz, Bergkamen; Prof. Dr. med. N. Sönnichsen, Berlin; Prof. Dr. med. W. Wohlrab, Halle; Prof. Dr. med. W.-I. Worret, München; Prof. Dr. med K.-D. Wozniak, Halle

\section{Verlag}

Urban \& Vogel GmbH, Aschauer Straße 30,

81549 München, Tel.: (0 89) 2030 43-13 00,

Fax: -13 99, www.springerfachmedien-medizin.de

\section{Inhaber- und Beteiligungsverhältnisse}

Die Urban \& Vogel GmbH ist 100\%ige

Tochtergesellschaft der Springer Medizin-Verlag $\mathrm{GmbH}$, Heidelberg. Die alleinige Gesellschafterin der Springer Medizin Verlag GmbH ist die Springer-Verlag GmbH mit einer Beteiligung von $100 \%$. Die Springer-Verlag GmbH ist eine 100\%ige Tochtergesellschaft der Springer Science + Business Media Deutschland GmbH Die alleinige Gesellschafterin der Springer Science + Business Media Deutschland $\mathrm{GmbH}$ ist die Springer Science + Business Media Netherlands B.V., die 100\% der Anteile hält. Die Springer Science + Business Media Netherlands B.V. ist eine 100\%ige Tochtergesellschaft der Springer Science+Business Media Finance S. à R. L.
Die Springer Science+Business Media Finance S. à R. L. ist eine $100 \%$ ige Tochter der Springer Science+Business Media S. A.

\section{Geschäftsführe}

Stephan Kröck, Harm van Maanen, Dr. Esther Wieland, Matthias Wissel

Director Facharzt Medizin

Dr. med. Christoph Posch (cp), verantwortlich i.S.d. Pressegesetzes

\section{Ressortleitung Dermatologie}

Markus Seidl

\section{Anzeigenleitung}

Sabine Weidner, Tiergartenstraße 17,

69121 Heidelberg

Tel. (0 62 21) 4 87-81 06, Fax: -87 62

E-Mail: sabine.weidner@springer.com

Anzeigenpreisliste Nr. 33 vom 1.10.2010

\section{Corporate Publishing}

Ulrike Hafner (Leitung), Tiergartenstraße 17, 69121 Heidelberg

Tel. (0 62 21) 4 87-81 04; Fax -6 8104

E-Mail: ulrike.hafner@springer.com

\section{Herstellung}

Alison Hepper (Leitung), Tel. (0 62 21) 4 87-82 65

E-Mail: alison.hepper@springer.com

Gabi Kellner (Layout)

\section{Vertrieb}

Frank Niemann (Leitung), Tel. (0 89) 2030 43-14 11

E-Mail: frank.niemann@springer.com

Druck

Kliemo Printing, Hütte 53, 4700 Eupen, Belgien

Die Zeitschrift wird auf chlorfrei gebleichtem Papier gedruckt.

\section{Abonnement}

hautnah dermatologie" erscheint sechsmal im Jahr. Bestellungen nimmt der Verlag unter Tel. (0 62 21) 3 45-43 04, Fax (062 21) 3 45-42 29 und www.springermedizin.de sowie jede Buchhandlung entgegen.

Das Abonnement gilt zunächst für ein Jahr. Es verlängert sich automatisch um jeweils ein weiteres Jahr, wenn dem Verlag nicht zwei Monate vor Ende des Bezugszeitraums die Kündigung vorliegt. Bezugspreise: Einzelheft 24,- $€$ inkl. MwSt. zzgl. Versand, Jahresabonnement 89,- $€$ inkl. deutscher MwSt. zzgl. Versand (ermäßigter Bezugspreis
z.B. für Studenten gegen Nachweis: 74,- $€$ ). Versand Inland: $21,-€$ Ausland $36,-€$.

\section{Copyright und Allgemeine Hinweise}

Zur Veröffentlichung kommen nur Arbeiten und Beiträge, die an anderer Stelle weder angeboten noch erschienen sind. Die Autoren sind verpflichtet zu prüfen, ob Urheberrechte Dritter berührt werden. Eine redaktionelle Bearbeitung bleibt vorbehalten. Für unverlangt eingesandte Manuskripte und Fotos wird keine Haftung übernommen. Der Verlag behält sich das ausschließliche Recht der Verbreitung, Übersetzung und jeglicher Wiedergabe auch von Teilen dieser Zeitschrift durch Nachdruck, Fotokopie, Mikrofilm, EDV-Einspeicherung, Funk- oder Fernsehaufzeichnung vor. Der Verlag kann den Beitrag auch online Dritten zugänglich machen (Online-Recht) und auf Datenträgern (CD-ROM etc.) verwerten (Offline-Recht). Jede gewerblich hergestellte oder benutzte Fotokopie verpflichtet nach Paragraph 54 (2) UrhRG zur Gebührenzahlung an die VG Wort, Abt. Wissenschaft,

Goethestr. 49, 80336 München, von der die Modalitäten zu erfragen sind.

Die Zeitschrift und alle in ihr enthaltenen Beiträge und Abbildungen sind urheberrechtlich geschützt. Mit Ausnahme der gesetzlich zugelassenen Fälle ist eine Verwertung ohne Einwilligung des Verlages strafbar.

Die Wiedergabe von Gebrauchsnamen, Handelsnamen, Warenbezeichnungen usw. in dieser Zeitschrift berechtigt auch ohne besondere Kennzeichnung nicht zu der Annahme, dass solche Namen im Sinne der Warenzeichen- und Markenschutzgesetzgebung als frei zu betrachten wären und daher von jedermann benutzt werden dürfen.

Für Angaben über Dosierungsanweisungen, Anwendungsgebiete und Applikationsformen von Medikamenten sowie für Abrechnungshinweise kann vom Verlag keine Gewähr übernommen werden. Derartige Angaben müssen vom jeweiligen Anwender im Einzelfall anhand anderer Literaturstellen auf ihre Richtigkeit überprüft werden.

(c) Urban \& Vogel GmbH 2011

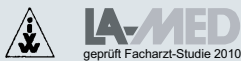

